University of Cambridge \& Cambridge University Hospitals NHS Trust

ks339@cam.ac.uk

Cite this as: BMJ 2021;375:n2680

http://dx.doi.org/10.1136/bmj.n2680

Published: 04 November 2021

\title{
Whole genome sequencing helps pinpoint a genetic diagnosis for patients
}

\section{Katherine Schon clinical research fellow and clinical genetics specialist registrar}

When I started as a specialty registrar in clinical genetics in 2013, genetic testing for rare disorders in the NHS involved single gene tests. These were performed at different specialist laboratories, by the labour-intensive process of Sanger sequencing. Due to the financial cost, sequencing was only ordered through clinical genetics departments, and requests were scrutinised at a departmental meeting. This approach could be successful for patients whose clinical features were highly specific for a single disorder, but for patients whose clinical features could be caused by mutations in many different genes (such as intellectual disability or mitochondrial disorders), finding a diagnosis by Sanger sequencing was extremely challenging.

Next generation sequencing allows hundreds or thousands of genes to be sequenced in a short period of time at a much lower cost. Whole exome sequencing (which looks at the DNA sequence for the protein coding parts of all genes) had been starting to become available for highly selected patients through research studies since around 2010, and I offered this enthusiastically to eligible patients. The 100000 Genomes Project had been announced in late 2012 with the aim of sequencing 100 ooo genomes from NHS patients with rare diseases or cancer, to benefit patients and introduce genomic medicine into the NHS.

The 100 ooo Genomes Project was striking for its scale and ambition. Patients with a large range of rare disorders could be enrolled, and the project also encouraged the mainstreaming of genetic testing by welcoming referrals from all hospital specialties. The UK was the first country in the world to announce such an extensive programme. After a pilot study, the main programme of the 100 ooo Genomes Project recruited patients from 2015 to 2018. Our hospital offered recruitment to the 100000 Genomes Project to patients for whom the standard NHS testing had not found a diagnosis.

When I started doing $\mathrm{PhD}$ research, working with the 100 ooo Genomes Project data seemed like an obvious choice. We focused on families who were recruited with a suspected mitochondrial disorder. Mitochondrial disorders are challenging to diagnose because they can be caused by mutations in nuclear-encoded genes or the mitochondrial DNA, and they can cause very diverse clinical presentations. The 100000 Genomes Project offers researchers a rich (pseudonymised) dataset about each participant, including their clinical presentation, their whole genome sequence, and a list of "tiered" variants (rare genetic variants which have been classed according to the likelihood that they are disease-causing for this participant). While looking at the data available, we developed our research questions. In how many families with suspected mitochondrial disorders can we find a genetic diagnosis? What sort of diagnoses do they have? What factors affect whether a diagnosis is found?

Two main challenges for our research emerged. One was finding bioinformatics support, although we managed to involve fantastic bioinformaticians from our team and collaborators. Our other difficulty was in contacting the referring clinicians and in feeding back potential new diagnoses. Systems were in place for both, but researchers are not able to contact clinicians directly and we were not able to make contact with the clinician for every family where it would be useful. The reasons for this were not always clear-perhaps it was due to official emails being missed or staff turnover. The system for feeding back new diagnoses was relatively slow in some instances, and did not provide the researcher with any feedback from the referring clinical centre. It would be helpful to develop closer integration between clinicians, bioinformaticians, clinical scientists, and academic research, as described in one centre in Sweden. ${ }^{1}$

The surprising finding from our study (doi:10.1136/BMJ-2021-066288) was that the number of mitochondrial diagnoses was lower than the number of other genetic diagnoses, including developmental disorders with intellectual disability, epileptic encephalopathies, cardiomyopathies, other metabolic disorders, and leukodystrophies. ${ }^{2}$ These non-mitochondrial disorders would have been missed if a traditional, targeted approach had been taken, and some had treatment implications. This highlights that the clinical diagnosis of rare genetic disorders is difficult, and that as clinicians we need to keep an open mind about the underlying diagnosis.

Making a genetic diagnosis is important for patients because it allows personalised advice about treatment, prognosis, and has implications for other family members or future children. We recommend that whole genome sequencing should be used early in the "diagnostic odyssey," before invasive tests such as muscle biopsy, so that patients can benefit from the power of this new technology.

\section{Competing interests: see linked Research (doi:10.1136/BMJ-2021-066288).}

Stranneheim H, Lagerstedt-Robinson K, Magnusson M, etal. Integration of whole genome sequencing into a healthcare setting: high diagnostic rates across multiple clinical entities in 3219 rare disease patients. Genome Med 2021;13:40. doi: 10.1186/s13073-021-00855-5. pmid: 33726816

Schon KR, Horvath R, Wei W, etal. Use of whole genome sequencing to determine genetic basis of suspected mitochondrial disorders: cohort study. BMJ2021;375:e066288. doi: 10.1136/BMJ-2021-066288. 